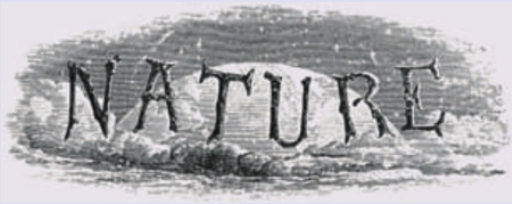

100 YEARS AGO

The columns of daily papers have during the last two weeks contained many references to the question of the origin of life... Prof. Ray Lankester and Dr. Chalmers Mitchell... proclaim themselves, as followers of Huxley, believers in evolution generally, and in the natural origin of living matter in the past. They, like many others, refuse to believe that it takes place at the present time, because undoubted proof of its occurrence cannot be produced by laboratory experiments. The uniformity of natural phenomena would certainly lead us to believe, as Sir Oliver Lodge has intimated, that if such a process occurred in the past, it should have been continually occurring ever since - so long as there is no evidence to show cause for a break in the great law of Continuity. Certainly no such evidence has ever been produced, and if the origin of living matter takes place by the generation in suitable fluids of the minutest particles gradually appearing from the region of the invisible, such a process may be occurring everywhere in nature's laboratories, though altogether beyond the ken of man. From Nature 10 November 1904.

\section{YEARS AGO}

Prof. Max Born, who has been awarded a share of the Nobel Prize in Physics for 1954, is known for many contributions to modern theoretical physics, particularly to the development of quantum mechanics and to the theory of crystals. The work mentioned specifically in the announcement of the Nobel award is the interpretation of the wave functions as probabilities for the positions of particles, a vital step in the development of the modern view of the relations between particle and wave aspects of atomic theory.

Prof. W. Bothe, of the University of Heidelberg, who shares the 1954 Nobel Prize in Physics with Prof. Born, is known for many important contributions to modern physics. The best known of these include his introduction of coincidence methods into counting techniques and the work in which, together with Geiger, he applied the coincidence method to the Compton effect and showed that the conservation laws are satisfied in each individual event and not merely on the average. This was fundamental for the interpretation of atomic processes.

From Nature 13 November 1954.
2. Houghton, J. T. et al. (eds) in Climate Change 2001: The Science of Climate Change (Cambridge Univ. Press, 2001).

3. Payette, S., Filion, L., Delwaide, A. \& Begin, C. Nature 341, 429-432 (1989).

4. Jarvis, P. \& Linder, S. Nature 405, 904-905 (2000).

5. Vaganov, E. A., Hughes, M. K., Kirdyanov, A. V., Schweingruber,

F. H. \& Silkin, P. P. Nature 400, 149-151 (1999).

6. Lennon, J. J., Kunin, W. E., Corne, S., Carver, S. \&
Van Hees, W. W. S. Glob. Ecol. Biogeogr. 11, 103-114 (2002).

7. Kullman, L. J. Ecol. 90, 68-77 (2002)

8. Körner, Ch. \& Paulsen, J. J. Biogeogr. 31, 713-732 (2004).

9. Loya, W. M. \& Grogan, P. Nature 431, 406-408 (2004).

10. Wookey, P. A. \& Robinson, C. H. Opera Botanica 132, 215-232 (1997)

11. Crawford, R. M. M. \& Abbott, R. J. Botanica Acta 107, 271-278 (1994)

Animal behaviour

\title{
Rank crime and punishment
}

\author{
Joan E. Strassmann
}

In paper wasps, facial markings are cheap 'status badges' that would seem to be susceptible to cheating. But wasps punish those whose markings lie. Social competition is, it appears, a strong selective force.

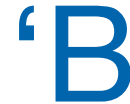
adges of status' are markings that animals are thought to use to signal their size and dominancethey are indicators of rank. To be useful, however, they must be 'honest' indicators; a symbol of high rank cannot be adopted by a low-quality individual. How can such cheating be prevented? The idea that badges of status must necessarily be honest because they are simply too costly for inferior individuals to produce has gained little experimental support. But there is a theory that social costs might instead be imposed on cheaters. On page 218 of this issue, Tibbetts and Dale ${ }^{1}$ provide support for this hypothesis. They show that in female paper wasps (Polistes dominulus), highly variable facial marks are related to overall size, and are used as rank indicators. Moreover, individuals painted with a mark that indicates a higher rank than they came by naturally are punished - something that has been difficult to demonstrate in other systems.

A century ago, Wilhelmine Enteman ${ }^{2}$ painstakingly documented within-colony colour variation in the social wasp Polistes. The purpose of such variation was unclear - communication was a possible function, but this was not investigated at the time. Subsequent work on communication among social insects instead focused on variation in nest and wasp chemicals. In wasps it was found, for instance, that kin recognition is based on variations in non-volatile, longchain hydrocarbons found on the insects' surface $^{3}$. Recently, however, Tibbetts ${ }^{4}$ reexamined the visual colour marks and found that they are important in communication after all.

Social wasps need to be able both to distinguish between individuals and to recognize the individuals' status, in order to establish and maintain a hierarchy of dominance. Mated females begin new nests together, and the winner of dominance contests lays most of the eggs. This dominance is usually expressed in low-cost ritualized postures rather than serious fighting ${ }^{5}$.
Tibbetts ${ }^{4}$ found that Polistes fuscatus females use individual identity cues based on facial and abdominal marks to recognize others in the hierarchy. She also showed that females become aggressive towards known females with altered marks.

Now, Tibbetts and Dale ${ }^{1}$ have examined the markings on P. dominulus females to see whether they are used as status badges. A status badge is a detectable indicator of rank that is related to a measure of competitive ability $^{6,7}$. Compared with individual identity cues, status badges are likely to be less variable, more dependent on physical condition and less strongly genetically determined ${ }^{8}$. The idea of badges of status was first invoked to explain rank-correlated variation in colour patterns among feeding flocks of birds that have frequent dominance interactions involving greater numbers of individuals than the birds could easily learn to identify individually ${ }^{6}$. A status badge would allow unfamiliar individuals to quickly determine, without aggression, who should give way. The concept has also been applied to territorial contests in which predicting the likelihood of losing would be advantageous ${ }^{7}$.

Polistes dominulus has highly variable dark spots on the clypeus (the region just above the mouth; Fig. 1). As Tibbetts and Dale describe ${ }^{1}$, spot number and brokenness - a measure that includes the number and irregularity of the spots — are slightly greater in wasps with wider heads. Head width is a well-known correlate of overall size and dominance in wasps. Thus the authors found, as predicted, that females with more spots or a higher brokenness index were more likely to become dominant in two-player contests.

A major question about colour-based status badges is why they do not seem to be vulnerable to cheating, even though they would appear to be 'cheap' to make. One hypothesis invokes social costs: an impostor would have to engage in more dominance contests, but would be likely to lose because of poor competitive ability. Support for this 
hypothesis has been equivocal in other systems, but now appears clear in $P$. dominulus. Losers were more likely to suffer continuing aggression from the dominant female if they had a more broken (higher-rank) pattern.

Going beyond correlation, Tibbetts and Dale manipulated the clypeus spots with yellow and black paint. To one member of a pair that had not previously interacted, the authors either added a spot, obliterated an existing spot, or added paint in ways that did not change the existing pattern. After these females had established a clear dominance relationship, dominant females continued to be aggressive towards subordinates whose painted marks indicated an increase in status. The result clearly supports the hypothesis that there are social costs to erroneously high status signals: cheating subordinates are punished.

The study had several puzzling features, however. The key result discussed above relates to behaviour after dominance had been established. Surprisingly, though, manipulating the spot pattern had no effect on behaviour before rank was established. Also, females painted with high-status marks were no more likely to attain dominant status than were those painted with lowstatus marks, or controls. If extra marks give a badge of high status, then their owner should be more likely to become dominant.

Another curiosity is that after dominance was established, the dominant females were also more aggressive towards subordinates painted to look even more subordinate. This is hard to explain in a two-female system. But wasps often begin nests in groups of more than two. A female with marks indicating very low rank that acted instead like a second-ranked female could plausibly be perceived by the dominant female as a threat and receive more aggression. A further unpredicted result is that unpainted subordinates were aggressive towards painted dominants, whether they were painted with a higher- or a lower-status mark than that of their 'true' status. In ten cases, the unpainted subordinate female even overthrew the painted dominant; these cases were evenly divided between those in which the dominant was painted with a higher- or a lowerstatus mark.

Also interesting is that the correlation of spot number or brokenness with head width is very weak, accounting for only $7 \%$ or $3 \%$ of the variance, respectively. A reliable cue of condition should account for more of the variance. Perhaps head width is not the best measure of condition, and perhaps the clypeus marks are actually correlated with a better, as-yet-unknown measure. But the marks are fixed in the pupal stage, and are not altered by later feeding and overwintering, which are known to affect condition. It seems that if clypeus marks are status badges, they are not conventional ones.

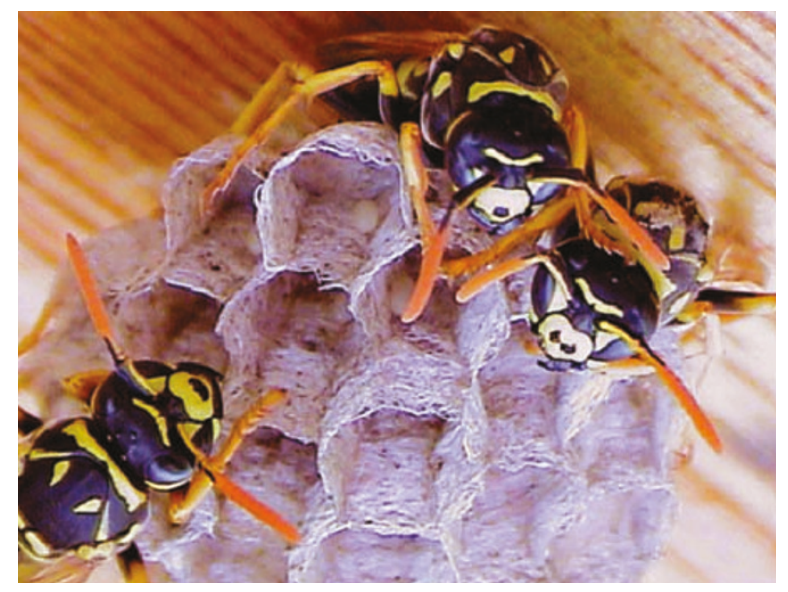

Figure 1 Badge of status. Polistes dominulus wasps show variation in the number and brokenness of spots on the clypeus, above the mouth. Tibbetts and Dale ${ }^{1}$ find that these measures provide an indicator of how dominant or subordinate an individual is; dishonesty brings social costs.

Tibbetts and Dale were careful to control for numerous factors that might have influenced the outcome of the contests. First, they paired wasps for mass. Second, they paired individuals that were unrelated and came from sites kilometres apart, so they could not have had any prior interactions. (In this species, females begin nests with relatives or nearby non-relatives with whom they are likely to have previously interacted ${ }^{9}$.) A further reason why the paired individuals could not have benefited from recognizing each other is that they were videotaped immediately after pairing. However, it is possible that they normally use individual recognition as well as status badges, as individual recognition can occur, at least in another Polistes species ${ }^{4}$.
It might seem remarkable that wasps have such sophisticated abilities. But social competition is a very strong selective force ${ }^{10}$. Pinyon jays can infer rank by watching others interact $^{11}$. Viruses can cheat ${ }^{12}$. Perhaps it should be no surprise that social wasps can recognize individuals and their rank.

Joan E. Strassmann is in the Department of Ecology and Evolutionary Biology, Rice University, Houston, Texas 77251-1892, USA.

e-mail:strassm@rice.edu

1. Tibbetts, E. A. \& Dale, J. Nature 432, 218-222 (2004)

2. Enteman, W. M. Carnegie Inst. Wash. Publ. 19, 1-88 (1904).

3. Singer, T. L., Espelie, K. E. \& Gamboa, G. J. in Pheromone

Communication in Social Insects: Ants, Wasps, Bees, and Termites (eds Vander Meer, R. K., Breed, M. D., Espelie, K. E. \& Winston, M. L.) 104-125 (Westview, Boulder, CO, 1998).

4. Tibbetts, E. A. Proc. R. Soc. Lond. B 269, 1423-1428 (2002).

\section{Plant development}

\section{A bunch of leaves}

The intricate and elegant architecture of a flower requires the activity of a plethora of proteins. Many belong to a group of gene regulators known as MADS-box proteins. These form multiprotein complexes that direct the formation of concentric whorls of sepals, petals, stamens and carpels. Gary Ditta et al. now show that without the concerted encouragement of four such proteins, all that is produced are clusters of leaves (Curr.

Biol. 14, 1935-1940; 2004).

The model plant Arabidopsis thaliana has more than a hundred genes for MADS-box proteins. Mutations in some of them produce dramatic effects whereas others have redundant functions requiring double, triple or even more mutants to uncover their roles. The SEPALLATA (SEP) family fall into this latter category, and earned their name when triple mutants - in which three members of the family were all disrupted — produced flowers made entirely of sepals. With this new work, a fourth sibling, SEP4, emerges.

Ditta et al. found that mutating SEP4 alone had no obvious effect, but quadruple mutants lacking all four SEP proteins formed 'flowers' with no recognizable floral organs (as shown here). Closer inspection revealed epidermal cells with the same shape and arrangement as in normal leaves. The presence of branched, hair-like structures called trichomes, a feature of Arabidopis leaves, put this

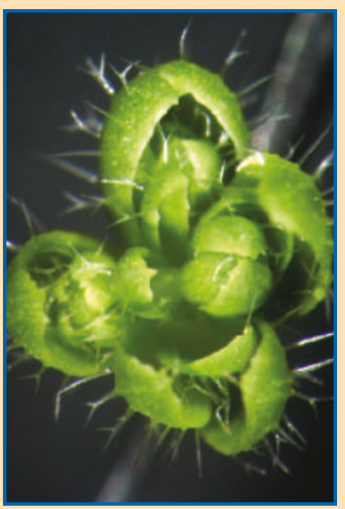

identification beyond doubt. In his treatise of 1790 , Die Metamorphose der Pflanzen (The Metamorphosis of Plants), the poet and philosopher Johann Wolfgang von Goethe proposed that flowers were modified leaves. Two hundred years later, stripping away the influence of the SEP proteins has returned them to their foliar state. Christopher Surridge 
5. Pardi, L. Physiol. Zool. 21, 1-13 (1948).

6. Rohwer, S. Evolution 29, 593-610 (1975).

7. Senar, J. C. in Proc. 22 Int. Ornithol. Congr. (eds Adams, N. J. \& Slotow, R. H.) 1669-1686 (1999).

8. Dale, J., Lank, D. B. \& Reeve, H. K. Am. Nat. 158, 75-86 (2001)
9. Queller, D. C. et al. Nature 405, 784-787 (2000)

10. West-Eberhard, M. J. Proc. Am. Phil. Soc. 123, 222-234 (1979).

11. Paz-y-Miño C, G., Bond, A. B., Kamil, A. C. \& Balda, R. P. Nature 430, 778-781 (2004).

12. Turner, P. E. \& Chao, L. Nature 398, 441-443 (1999).

Condensed-matter physics

\section{A magnetic pendulum}

\section{Claude Chappert and Thibaut Devolder}

Where two oppositely magnetized regions meet, there is a so-called domain wall. Under the right conditions, this wall can be made to oscillate like a pendulum, suggesting a new approach to electronics.

$\mathrm{O}$ n page 203 of this issue, Saitoh et al. ${ }^{1}$ provide a beautiful demonstration of a magnetic domain wall oscillating just as a pendulum would do under gravity. A domain wall separates two uniformly magnetized regions and is predicted, at low excitation energies, to behave like a particle with a finite mass. By trapping a single magnetic domain wall in a strip of ferromagnetic material, Saitoh et al. show that they can force the oscillations of the wall inside this potential well, achieving resonant displacements of micrometre length at megahertz frequencies - all with only moderate currents, a key result for possible applications.

The experiment designed by Saitoh et al. is very clever. They patterned a narrow semicircular loop of $\mathrm{Ni}_{81} \mathrm{Fe}_{19}$ wire, $70 \mathrm{~nm}$ wide and $45 \mathrm{~nm}$ deep, onto a silicon substrate. In such a wire of soft ferromagnetic material, dipolar interactions tend to maintain the magnetization parallel to the wire edges. A domain wall will thus separate two areas that are uniformly magnetized antiparallel to each other (Fig. 1a). These 'head-to-head' walls have a nanoscale core that is magnetized perpendicular to the local wire direction. And this is where the semicircular shape comes into play: a magnetic field strong enough to saturate the magnetization of the whole loop, when reduced again to zero, will either leave a single wall in the loop or destroy a pre-existing one, depending solely on the field's orientation (Fig. 1b,c). If a wall is created, increasing the field back to moderate values will tend to trap the wall at the position that keeps its core magnetized parallel to the field direction. The wall will then behave in a manner similar to a simple pendulum, which relaxes towards a stable position under gravity. But here the role of gravity is taken by the magnetic field and hence can be tuned in amplitude and direction gravity itself is not so easy to manipulate!

Applying a periodic force to this'magnetic pendulum' results in an oscillation, whose resonant frequency depends on the effective 'mass' of the domain wall: Saitoh et al. have determined that mass to be $6.6 \times 10^{-23} \mathrm{~kg}$.

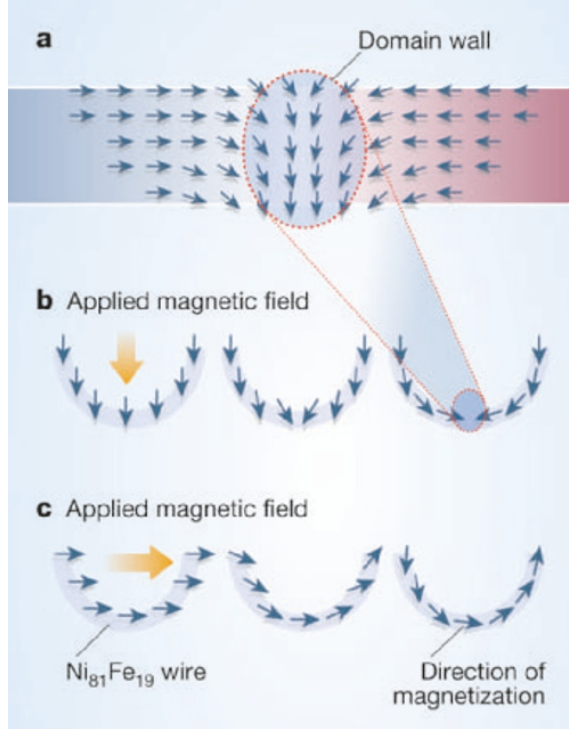

Figure 1 A magnetic pendulum in tunable gravity. a, Where two oppositely magnetized regions meet, there is a domain wall. The arrows indicate the direction of magnetization. Saitoh et al. ${ }^{1}$ have explored the magnetization in a semicircular loop of $\mathrm{Ni}_{81} \mathrm{Fe}_{19}$ wire. $b$, When a magnetic field is applied as shown and then removed, the magnetization of the wire settles into a configuration that includes a single domain wall. c, A magnetic field applied in a perpendicular orientation, however, sets the magnetization such that there is no domain wall. When a domain wall is created, Saitoh et al. have shown that it can be made to behave like a pendulum does under gravity, although in this case the 'gravitational' force - the strength and direction of the magnetic field — can be tuned across a range of values.

The authors have gone further, to investigate the oscillation triggered by passing a sinusoidal current through the wire, using its resonance to explore the interaction between the electrons and the wall. Two types of competing interactions are expected. One is 'momentum transfer', which is basically the mechanical pressure exerted by electrons reflected from the domain wall. The other is 'spin transfer', which arises from the fact that the spins of electrons passing through the domain wall rotate: because total angular momentum is conserved, angular momentum is transferred from the electrons to the magnetization itself, which can trigger movement of the domain wall.

To identify which mechanism is the more relevant, Saitoh et al. take advantage of the different frequency-dependent efficiencies of each transfer process. They find that momentum transfer is a hundred times more efficient than spin transfer in the frequency range of this experiment (from a few to 75 megahertz). This is unexpected in ferromagnetic metals, such as the nickeliron compound used here: most scientists believed that domain walls in such materials are wide enough for flowing spins to rotate adiabatically and track the local magnetization orientation, leading to the dominance of spin transfer. Moreover, by properly choosing the excitation frequency, the threshold current to displace the domain wall could be reduced to a few times $10^{9} \mathrm{~A} \mathrm{~m}^{-2}$, a hundredth of what is observed with direct currents, and well below any heating threshold.

Magnetic domain walls can be considered to be self-assembled stable nanostructures; moreover, they can be created or annihilated by external action. The manipulation of domain walls in stripes has already been proposed as a way of storing information or even performing logic functions ${ }^{2}$. With their result, Saitoh et al. ${ }^{1}$ now provide a low-energy handle on the manipulation of domain walls in complex circuit architectures. And this technique can be fast ${ }^{3}$ and is fairly scalable - the only requirement is to maintain a constant current density as the stripe dimensions are reduced. Saitoh et al. propose some rules of thumb to reduce the required current density even further. In fact, if the ferromagnetic metal were replaced by a dilute magnetic semiconductor, the current density needed to move a wall would already be much smaller ${ }^{4}$.

Conventional (CMOS) electronics is facing major technical hurdles, particularly that of miniaturization. New approaches are needed to reduce energy dissipation and fabrication costs while maintaining the rate of reduction of dimensions, and must be implemented within 15-20 years. This work ${ }^{1}$ could well find application in this direction. Saitoh and colleagues could be a step closer to a new'domain-wall' electronics.

Claude Chappert and Thibaut Devolder are at the Institut d'Electronique Fondamentale,

UMR CNRS 8622, Université Paris-Sud,

91405 Orsay cedex, France.

e-mail: claude.chappert@ief.u-psud.fr

\footnotetext{
1. Saitoh, E., Miyajima, H., Yamaoka, T. \& Tatara, G. Nature 432, 203-206 (2004)

2. Allwood, D. et al. Science 296, 2003-2006 (2002).

3. Nakatani, Y., Thiaville, A. \& Miltat, J. Nature Mater. 2, 521-523 (2003).

4. Yamanouchi, M., Chiba, D., Matsukura, F. \& Ohno, H. Nature
} 428, 539-542 (2004) 